\title{
Exercise Echocardiography for the Prediction of Major Cardiac Events and Overall Mortality after Coronary Artery By-Pass Surgery
}

\author{
Jesús Peteiro ${ }^{1 *}$, Alberto Bouzas-Mosquera1, Dolores Martinez ${ }^{1}$, Jose M Vazquez-Rodriguez ${ }^{1}$ and \\ Francisco Broullon ${ }^{2}$
}

${ }^{1}$ Department of Cardiology and Information Technology, Hospital Universitario de A Coruña (CHUAC), A Coruña, Spain

${ }_{2}^{2}$ Institute of Biomedical Research of a Coruña (INIBIC), and University of a Coruña. A Coruña, Spain

Received: January 21, 2018; Published: February 01, 2018

*Corresponding author: Jesús Peteiro, Department of Cardiology, Hospital Universitario de A Coruña, (CHUAC), A Coruña, Spain, Tel: 34 981917859; Email: pete@canalejo.org

Abstract

Objective: There is limited information about the value of exercise echocardiography for the prediction of events after coronary artery by-pass surgery (CABS). Ischemia might be considered of no much value in patients already revascularized by surgery. We sought to investigate the value of exercise echocardiography (ExE) for the prediction of major cardiac events (MACE) and overall mortality in patients submitted to CABS.

Methods: Retrospective analysis of prospectively collected data on 568 CABS patients (mean age $66 \pm 10$ years) who were submitted to a clinically indicated first treadmill ExE. Normal ExE was defined as the absence of wall motion abnormalities (WMAs) at rest and at exercise. Ischemia was defined as the appearance of new WMAs or the worsening of resting WMAs. Abnormal ExE was defined as ischemia or the presence of resting WMAs unchanged with exercise. The end points were MACE and overall mortality.

Results: During a mean follow-up of 6.6 \pm 5.4 years, there were 132 MACE before any subsequent revascularization and 196 deaths. An abnormal ExE was observed in 432 patients (76\%) and ischemia in 274 (48\%). After multivariate adjustment that included clinical characteristics, resting left ventricular function and exercise ECG testing results, ischemia during ExE was an independent predictor of MACE (Hazard ratio $[\mathrm{HR}]=1.64,95 \%$ Confidence Interval $[\mathrm{CI}]=1.15-2.34, \mathrm{p}=0.006)$, and a marginally predictor of overall mortality $(\mathrm{HR}=1.34,95 \%$ Confidence Interval $[\mathrm{CI}]=1.00-1.78, \mathrm{p}=0.05)$. Other predictors of MACE were resting left ventricular ejection fraction and maximal achieved workload in metabolic equivalents. Annualized MACE rates were double in patients with ischemia in comparison to patients without ( $6.8 \%$ vs. $3.4 \%, \mathrm{p}<0.001)$, and quadruple in patients with abnormal ExE in comparison to those with a normal ExE (6.0\% vs. $1.9 \%, \mathrm{p}<0.001)$.

Conclusions: An abnormal ExE is prevalent among surgical revascularized patients with clinically indicated ExE, and is a predictor of events.

Keywords: Exercise echocardiography; by-pass surgery; ischemia

Abbreviations: BP: Blood pressure; CABS: Coronary artery by-pass surgery; CAD: Coronary artery disease; ECG: Electrocardiogram; ExE: Exercise echocardiography; LVEF: Left ventricular ejection fraction; MACE: Major cardiac events; MAPHR: Mean age-predicted heart rate; MI: Myocardial infarction; WMAs: Wall motion abnormalities; WMSI: Wall motion score index; RASS denotes renin-angiotensin-aldosterone system; IMA: internal mammary artery; METs denotes metabolic equivalents; RPP, rate pressure product (1000 mmHg x beats/min);

\section{Introduction}

Stress echocardiography has demonstrated its ability to unmask ischemia in different scenarios, including post revascularization of coronary artery disease (CAD) [1-4]. Although revascularization of CAD by coronary artery by-pass surgery (CABS) has been in part replaced by non surgical revascularization procedures [5]. these non-surgical procedures are in general more reserved for patients with less presence of multivessel coronary artery disease (CAD). On the contrary patients submitted to CABS usually have extensive multivessel $\mathrm{CAD}$ and complete revascularization is not always achievable, leading to a significant number of patients that may have ischemia under stress conditions. Also, the impact of symptoms progression and ischemia in these patients is a 
matter of concern and has been just partially evaluated by stress echocardiography. Ischemia might be considered of limited value for patients already revascularized by surgery, as subsequent revascularization procedures in them could be risky. In addition, although guidelines state the appropriateness of functional imaging in symptomatic patients after surgery of CAD, it is less clear its role in asymptomatic patients, at least within 5 years after revascularization [6]. The aim of this study was to investigate the value of exercise echocardiography (ExE) for the prediction of major cardiac events (MACE) and overall mortality in patients submitted to CABS.

\section{Methods}

Collected data from the University of A Coruña stress echocardiography laboratory databank on patients with previous CABS were retrospectively analyzed.

\section{Patients}

Data were obtained from 568 patients with previous CABS that underwent a treadmill ExE in our institution in a 19 year-period from March 1995 to December 2014. Data were extracted from a database of 18,031 cases, with a first ExE performed in 14,906 patients. Patients with moderate or severe valvulopathies $(n=55)$ and hypertrophic cardiomyopathy $(\mathrm{n}=21)$ were not included. Also, patients with previous CABS that underwent dobutamine stress echocardiography during the same period of time due to inability to exercise were also excluded $(n=46)$ Demographic and clinical data, as well as stress testing results, were entered in our database at the time of the procedures. Data were taken from the original ExE reports of the patients. Whenever possible, ß-blocker therapy was discontinued for at least 48 hours before testing. However, $21 \%$ of the patients were still under the influence of beta-blockers at the time of their tests.

\section{Exercise ECG Testing}

Heart rate, blood pressure (BP), and ECG were obtained at baseline and at each stage of exercise. Patients were encouraged to perform a maximal treadmill exercise test (Bruce protocol 87.5\%, Bruce protocol for fitness patients 2.6\%, modified Bruce 7.4\%, Naughton 2.5\%). Exercise end points included physical exhaustion, significant arrhythmia, severe hypertension (systolic BP $>240 \mathrm{~mm}$ $\mathrm{Hg}$ or diastolic BP $>110 \mathrm{~mm} \mathrm{Hg}$ ), severe hypotensive response (decrease $>20 \mathrm{~mm} \mathrm{Hg}$ ), or symptoms during exercise. Ischemic ECG was defined as the development of ST-segment deviation of $\geq 1 \mathrm{~mm}$ which was horizontal or down sloping away from the is electric line $80 \mathrm{~ms}$ after the J point in at least 2 leads, in patients with normal baseline ST segments. The ECG was considered non-diagnostic in the presence of left bundle branch block, preexcitation, paced rhythm, repolarization abnormalities or treatment with digoxin. Positive exercise testing was defined as chest pain during the test and/or ischemic ECG abnormalities in patients with diagnostic ECG (7-8). A maximal test was defined as the achievement of at least $85 \%$ of the mean age-predicted heart rate (MAPHR), otherwise the test was considered sub maximal. All patients gave informed consent.

\section{Exercise echocardiography and echocardiographic analysis}

Echocardiography was performed in 3 apical views (long axis, 4-, and 2-chambers) and 2 parasternal views (long- and shortaxis) at baseline, peak exercise [7-9] and in the immediate postexercise period. Peak exercise imaging on the treadmill has been previously described by our group $[9,10]$. It is performed with the patient still exercising, when signs of exhaustion are present or an end point is achieved. If the patient is running, she/he is asked to walk fast instead of running during acquisition. In addition it may be necessary to keep steady the velocity of the treadmill. The transducer is firmly positioned on the apical and then parasternal areas by applying pressure to the patient's back with the left hand, so maintaining the patient between the transducer and the left hand, in order to diminish body and respiratory movements. A continuous imaging acquisition system is used. We have demonstrated higher heart rates during acquisition with peak than with post exercise imaging, and also higher sensitivity and prognostic value [8-10]. Regional wall motion abnormalities (WMAs) were evaluated with a 16-segment model of the left ventricle [11]. Each segment was graded on a 4-point scale, with normal wall-motion scoring $=1$, hypokinetic $=2$, akinetic $=3$, dyskinetic $=4$, and non-visualized $=0$. However, isolated hypokinesia of the basal inferior or inferoseptal segments was not considered abnormal [12]. Wall motion score index (WMSI) and visually estimated left ventricular ejection fraction (LVEF) (13) were calculated at rest, peak and post-exercise.

WMSI was calculated as the sum of scores divided by the number of visualized segments. The worst WMSI and LVEF obtained at peak or post-exercise imaging were considered as definitive values. The change in WMSI from rest to exercise (WMSI) was calculated. Ischemia was defined as the development of new or worsening WMAs with exercise, and a fixed WMA as a WMA that persisted the same with exercise. The extension of ischemia was further graded as mild when it involved 1 to 2 segments, moderate when it involved 3 to 4 segments, and extensive when it involved $\geq 5$ segments. An abnormal ExE was defined as ischemia or fixed WMAs.

\section{Follow-Up and End-Points}

Follow-up in the entire study cohort of 568 patients was obtained by review of hospital databases, medical records and death certificates, as well as by telephone interviews when necessary. We had complete access to the electronic health care system of our community, and also electronic access to the GP consults. In case of death out of a hospital, the cause of death was provided by the Mortality Registry of our community. No patients were lost during follow-up. Considered end-points were overall mortality and major cardiac events (MACE). MACE was considered in case of cardiac death or non fatal MI. MI was defined as the appearance of new symptoms of myocardial ischemia or ischemic ECG changes accompanied by increases in markers of myocardial necrosis. Cardiac death was defined as death due to acute MI, congestive heart failure, life-threatening arrhythmias, or cardiac arrest. Unexpected sudden death without an identified cardiac cause was 
also considered cardiac death. Revascularization procedures during follow-up were not considered events as the ExE results might have influenced patient management. However, they were recorded, and the impact of subsequent revascularization on outcome assessed.

\section{Statistical Analysis}

Categorical variables were reported as \% and comparison between groups based on the $\chi 2$ test. Continuous variables were reported as mean \pm 1 standard deviation and intergroup differences were assessed with the unpaired Student $t$ test or the Mann-Whitney U test, as appropriate. A P value of $<.05$ was considered statistically significant. Patients were censored at the time of a coronary revascularization procedure for the analysis of MACE, but not for the analysis of overall mortality [14]. Survival free of the end point of interest was estimated by the Kaplan-Meier method, and survival curves were compared with the log-rank test. Annualized event rates were assessed according to the number of events, the number of patients at risk and the mean time of followup of the group at risk. Univariable and multivariable associations of the different variables with outcome were assessed with Cox's proportional hazard model. Variables were selected in a stepwise forward selection manner, with entry and retention set at $\mathrm{p}=0.05$. Hazard ratios (HR) with 95\% confidence intervals (CI) were estimated. The incremental value of ExE results over clinical, resting echocardiography and exercise treadmill testing variables was assessed in steps. The 1st step was based on clinical data. Resting echocardiography data and exercise ECG data were then added in the following step. The 3th step consisted of echocardiography data obtained during exercise. A statistically significant increase in the global $\chi 2$ defined incremental prognostic value [15]. Statistical analysis was performed using SPSS software, version 15.0 (SPSS, Chicago, IL).

\section{Results}

\section{Clinical baseline characteristics and tests results shown in Tables 1 and 2}

The clinical baseline characteristics and resting and exercise echocardiography results of the overall group are shown in (Table 1 and 2).

Table 1: Baseline clinical characteristics of the 568 patients.

\begin{tabular}{|c|c|}
\hline Age, years & $66 \pm 10$ \\
\hline Male gender, $\mathrm{n}(\%)$ & $479(84)$ \\
\hline Diabetes Mellitus, $\mathrm{n}(\%)$ & $143(25)$ \\
\hline Hypertension, $\mathrm{n}(\%)$ & $300(53)$ \\
\hline Hypercholesterolemia, $\mathrm{n}(\%)$ & $326(57)$ \\
\hline Smoking, $\mathrm{n}(\%)$ & $108(19)$ \\
\hline Atrial fibrillation, $\mathrm{n}(\%)^{*}$ & $32(6)$ \\
\hline Reasons for testing, $\mathrm{n}(\%)$ & \\
\hline Typical angina & $84(15)$ \\
\hline Atypical angina & $271(48)$ \\
\hline Non coronary chest pain & $59(10)$ \\
\hline
\end{tabular}

\begin{tabular}{|c|c|}
\hline Dyspnea & $16(3)$ \\
\hline $\begin{array}{c}\text { Asymptomatic/Post-surgical } \\
\text { evaluation }\end{array}$ & $138(24)$ \\
\hline Abnormal resting ECG, n (\%) & $169(30)$ \\
\hline \multicolumn{2}{|l|}{ Medications, n (\%) } \\
\hline Betablockers* & $119(21)$ \\
\hline RAAS system & $216(38)$ \\
\hline Nitrates & $239(42)$ \\
\hline $\mathrm{Ca}++$ antagonists & $91(16)$ \\
\hline Diuretics & $49(9)$ \\
\hline Digoxin & $19(3)$ \\
\hline $\begin{array}{l}\text { Months after surgery, median (25- } \\
\text { 75th percentiles)** }\end{array}$ & $58(20-120)$ \\
\hline in symptomatic patients & $60(24-120)$ \\
\hline in asymptomatic patients & $29(13-60)$ \\
\hline \multicolumn{2}{|l|}{ Type of surgery, n (\%) } \\
\hline unknown & $38(7)$ \\
\hline 2 IMA+1 to 2 vein grafts & $200(35)$ \\
\hline $1 \mathrm{IMA}+3$ vein grafts & $163(29)$ \\
\hline 1 to 2 vein grafts & $62(11)$ \\
\hline 3 vein grafts & $105(18)$ \\
\hline
\end{tabular}

Table 2: Resting and exercise echocardiography results.

\begin{tabular}{|c|c|}
\hline Achieved workload in METs & $8.3 \pm 3.1$ \\
\hline \% achieved of the MAPHR & $89 \pm 15$ \\
\hline Submaximal test, $\mathrm{n}(\%)$ & $214(38)$ \\
\hline Resting heart rate (bpm) & $76 \pm 16$ \\
\hline Resting blood pressure (mmHg) & $135 \pm 20$ \\
\hline Peak heart rate (bpm) & $137 \pm 23$ \\
\hline Peak blood pressure (mmHg) & $163 \pm 29$ \\
\hline $\begin{array}{c}\text { Peak double product (HR x BP x } \\
10(3))\end{array}$ & $22.6 \pm 6.1$ \\
\hline LVEF & $54 \pm 11$ \\
\hline at rest & $55 \pm 14$ \\
\hline at peak exercise & $1.25 \pm 0.32$ \\
\hline WMSI & $1.42 \pm 0.39$ \\
\hline at rest & $130(23)$ \\
\hline at peak exercise & $176(31)$ \\
\hline Positive exercise ECG, $n(\%)$ & $314(55)$ \\
\hline $\begin{array}{c}\text { Symptoms during exercise testing, } \\
\mathrm{n}(\%)\end{array}$ & $274(48)$ \\
\hline $\begin{array}{c}\text { Resting wall motion abnormalities, } \\
\mathrm{n}(\%)\end{array}$ & $432(76)$ \\
\hline Ischemia, $\mathrm{n}(\%)$ & \\
\hline Abnormal ExE, $\mathrm{n}(\%)$ & $(\%)$ \\
\hline
\end{tabular}

\section{Prediction of outcome}

Univariable and multivariable analysis of MACE and overall mortality are depicted in Tables 3 and 4, respectively. Ischemia was an independent predictor of MACE and a marginally predictor of overall mortality. When introduced instead of ischemia, all the 
other ExE variables were also predictive of MACE: peak WMSI (HR=3.29, 95\% CI=2.15-5.04, p<0.001), peak LVEF (HR=0.97, 95\% CI=0.96-0.98, p<0.001), $\Delta$ in WMSI $(\mathrm{HR}=2.62,95 \% \mathrm{CI}=1.33-$ 5.19, $\mathrm{p}=0.006)$, and $\Delta$ in LVEF (HR=0.96, 95\% CI=0.94-0.98, $\mathrm{p}=0.001$ ). Figure 1 shows the Chi-square of the models and their change with the addition of the ExE results for the prediction of MACE and overall mortality. Annualized MACE rates were double in patients with ischemia in comparison to patients without $(6.8 \%$ vs. $3.4 \%, \mathrm{p}<0.001$ ), and quadruple in patients with abnormal ExE in comparison to those with normal $\operatorname{ExE}(6.0 \%$ vs. $1.9 \%, \mathrm{p}<0.001)$. Similarly, annualized overall mortality rates were $5.8 \%$ in patients with abnormal ExE results and 3.7\% in patients with normal ExE results $(p=0.007)$, whereas the values for annualized mortality rates in patients with and without ischemia were $6.2 \%$ and $4.2 \%$, respectively ( $p=0.004)$. and Figures 2 and 3 show show the KaplanMeier curves for MACE and overall mortality in patients with and without ischemia and in those with and without abnormal ExE. Figure 4 shows MACE-free survival curves for patients with and without increase in LVEF with exercise, with and without ischemia associated to resting WMAs, and for patients classified according to the extension of ischemia. The worst annualized MACE rates were observed in patients with no increase in LVEF with exercise $(7.7 \%)$ in comparison to patients with increase in LVEF (3.5\%), in patients with ischemia associated to resting WMAs (7.2\%) in comparison to patients without these characteristics (3.9\%), and in patients with extensive ischemia. Annualized MACE rate in patients with extensive ischemia was $9.3 \%$, whereas it was $5.3 \%$ in patients with moderate ischemia, $6.2 \%$ in those with mild ischemia, and $3.4 \%$ in subjects without ischemia.

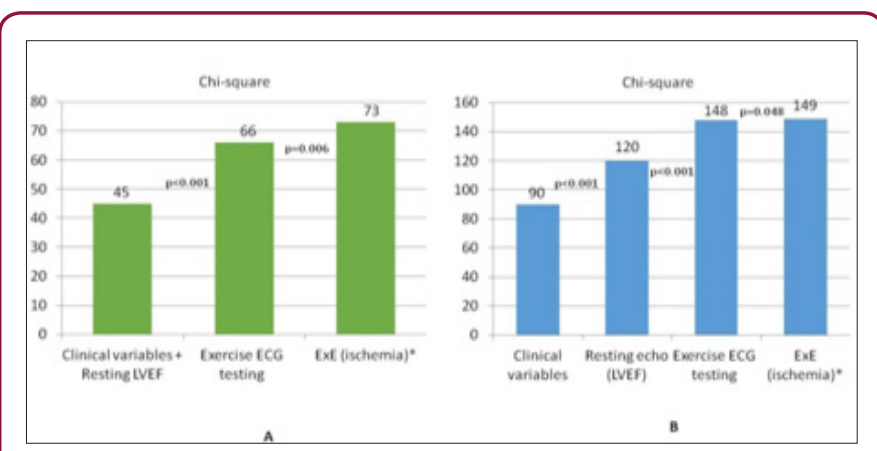

Figure 1: Chi-square of the models for the prediction of MACE (A) and of overall mortality (B)

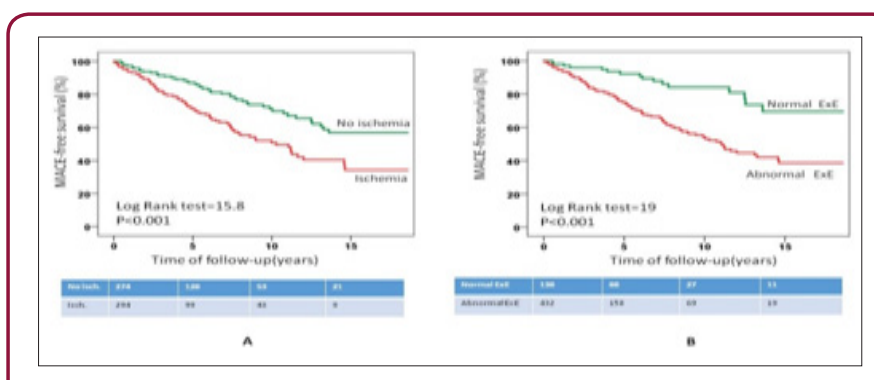

Figure 2: Kaplan-Meier curves for major cardiac events in patients with and without increase in LVEF with exercise, with and without ischemia associated to resting WMAs, and for patients classified according to the extension of ischemia.

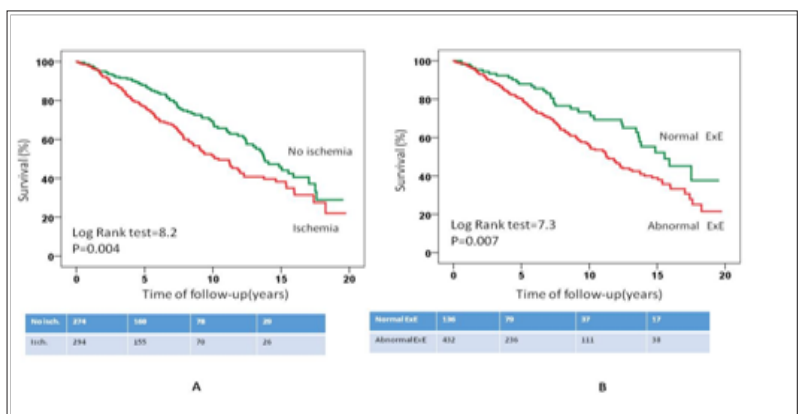

Figure 3: Kaplan Meier curves for overall mortality in patients with and with and without cischemia (A) and in patients with and without abnormal exercise echocardiography (B).

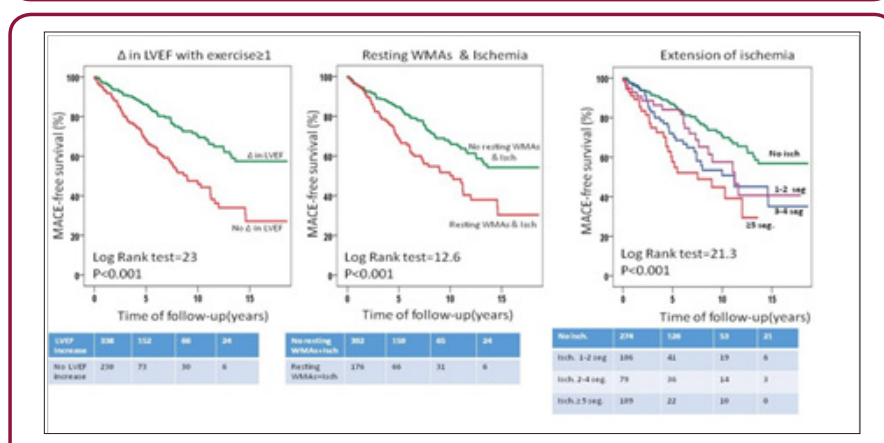

Figure 4: Kaplan Meier curves for major cardiac events in patients with and without ischemia (A) and in patients with and without abnormal exercise echocardiography (B).

Table 3: Univariable and multivariable predictors of major cardiac events

\begin{tabular}{|c|c|c|c|c|c|c|}
\hline \multirow{2}{*}{ Clinical variables } & \multicolumn{3}{|c|}{ Univariable } & \multicolumn{3}{|c|}{ Multivariable } \\
\hline & $H R$ & $95 \% C I$ & $P$ & $H R$ & $95 \% C I$ & $p$ \\
\hline Age (per year) & 1.03 & $1.01-1.05$ & 0.001 & & & \\
\hline Diabetes Mellitus & 1.48 & $1.00-2.17$ & 0.049 & & & \\
\hline $\begin{array}{c}\text { Abnormal resting } \\
\text { ECG }\end{array}$ & 1.73 & $1.23-2.44$ & 0.002 & & & \\
\hline Dyspnea & 2.48 & $1.09-5.66$ & 0.03 & & & \\
\hline RAAS blockers & 1.49 & $1.05-2.13$ & 0.03 & & & \\
\hline Diuretics & 2.03 & $1.28-3.21$ & 0.003 & & & \\
\hline \multicolumn{7}{|c|}{ Resting echocardiography } \\
\hline
\end{tabular}




\begin{tabular}{|c|c|c|c|c|c|c|}
\hline LVEF at rest & 0.96 & $0.95-0.97$ & $<0.001$ & 0.97 & $0.96-0.99$ & $<0.001$ \\
\hline WMSI at rest & 4.18 & $2.72-6.44$ & $<0.001$ & & & \\
\hline \multicolumn{7}{|l|}{$\begin{array}{l}\text { Exercise ECG } \\
\text { testing }\end{array}$} \\
\hline $\begin{array}{l}\text { Achieved workload } \\
\text { in METs }\end{array}$ & 0.83 & $0.78-0.88$ & $<0.001$ & 0.86 & $0.81-0.92$ & 0.001 \\
\hline$\Delta$ in RPP & 0.91 & $0.88-0.94$ & $<0.001$ & & & \\
\hline Peak RPP & 0.93 & $0.90-0.96$ & $<0.001$ & & & \\
\hline $\begin{array}{c}\% \text { achieved of the } \\
\text { MAPHR }\end{array}$ & 0.98 & $0.97-0.990$ & 0.002 & & & \\
\hline \multicolumn{7}{|c|}{ Peak exercise echocardiography } \\
\hline Peak WMSI & 4.37 & $2.88-6.62$ & $<0.001$ & & & \\
\hline Peak LVEF & 0.96 & $0.95-0.97$ & $<0.001$ & & & \\
\hline$\Delta$ in WMSI & 2.16 & $1.11-4.19$ & 0.02 & & & \\
\hline$\Delta$ in LVEF & 0.96 & $0.94-0.98$ & $<0.001$ & & & \\
\hline Ischemia & 2.01 & $1.42-2.86$ & $<0.001$ & 1.64 & $1.15-2.34$ & 0.006 \\
\hline Abnormal ExE & 3.02 & $1.79-5.09$ & $<0.001$ & & & \\
\hline
\end{tabular}

At the time of the ExE; **Data available for 430 patients. treatment with digoxin, history of typical angina, asymptomatic Other non significant analyzed variables were gender, smoking, status, atrial fibrillation at the time of the ExE, resting rate-pressure hypercholesterolemia, hypertension, family history of CAD, product, angina during exercise testing and positive exercise ECG treatment with beta-blockers at the time of the exercise testing, testing treatment with nitrates, treatment with calcium channel blockers,

Table 4: Univariable and multivariable predictors of overall mortality.

\begin{tabular}{|c|c|c|c|c|c|c|}
\hline \multirow{2}{*}{ Clinical variables } & \multicolumn{3}{|c|}{ Univariable } & \multicolumn{3}{|c|}{ Multivariable } \\
\hline & HR & $95 \% \mathrm{CI}$ & $\mathbf{P}$ & HR & $95 \% \mathrm{CI}$ & \\
\hline Age (per year) & 1.08 & $1.06-1.10$ & $<0.001$ & 1.06 & $1.04-1.08$ & $<0.001$ \\
\hline Diabetes Mellitus & 1.47 & $1.06-2.04$ & 0.02 & & & \\
\hline Dyspnea & 2.24 & $1.05-4.81$ & 0.04 & & & \\
\hline Atrial fibrillation* & 2.39 & $1.43-4.00$ & 0.001 & & & \\
\hline $\begin{array}{c}\text { Abnormal resting } \\
\text { ECG }\end{array}$ & 1.93 & $1.46-2.56$ & $<0.001$ & & & \\
\hline Digoxin & 2.3 & $1.31-4.05$ & 0.004 & & & \\
\hline Diuretics & 2.76 & $1.89-4.03$ & $<0.001$ & 1.7 & $1.14-2.53$ & 0.009 \\
\hline RAAS blockers & 1.36 & $1.01-1.83$ & 0.04 & & & \\
\hline \multicolumn{7}{|c|}{ Resting echocardiography } \\
\hline LVEF at rest & 0.96 & $0.95-0.97$ & $<0.001$ & 0.98 & 0.97-0.99 & $<0.001$ \\
\hline WMSI at rest & 3.67 & $2.49-5.40$ & $<0.001$ & & & \\
\hline \multicolumn{7}{|l|}{$\begin{array}{l}\text { Exercise ECG } \\
\text { testing }\end{array}$} \\
\hline $\begin{array}{c}\text { Achieved workload } \\
\text { in METs }\end{array}$ & 0.79 & $0.75-0.83$ & $<0.001$ & 0.84 & $0.79-0.89$ & $<0.001$ \\
\hline$\triangle$ in $\mathrm{RPP}$ & 0.91 & $0.89-0.94$ & $<0.001$ & & & \\
\hline Peak RPP & 0.93 & $0.91-0.96$ & $<0.001$ & & & \\
\hline Positive ECG & 0.61 & $0.41-0.89$ & 0.01 & & & \\
\hline \multicolumn{7}{|l|}{$\begin{array}{c}\text { Peak exercise } \\
\text { echocardiography }\end{array}$} \\
\hline Peak WMSI & 2.6 & $1.85-3.67$ & $<0.001$ & & & \\
\hline Peak LVEF & 0.97 & $0.96-0.98$ & $<0.001$ & & & \\
\hline
\end{tabular}




\begin{tabular}{|c|c|c|c|c|c|}
\hline$\Delta$ in LVEF & 0.98 & $0.97-1.00$ & 0.04 & & \\
\hline Ischemia & 1.51 & $1.14-2.01$ & 0.004 & 1.34 & $1.00-1.78$ \\
\hline Abnormal ExE & 1.65 & $1.14-2.38$ & 0.008 & & \\
\hline
\end{tabular}

Other non significant analyzed variables were gender, smoking, hypercholesterolemia, hypertension, family history of CAD, treatment with beta-blockers at the time of the exercise testing, treatment with nitrates, treatment with calcium channel blockers, history of typical angina, asymptomatic status, resting ratepressure product, \% achieved of the maximal age-predicted heart rate, and angina during exercise testing.

\section{Symptomatic versus asymptomatic patients}

A total of 138 patients were asymptomatic (24\%), whereas the rest have had clinical symptoms before the ExE, like typical or atypical chest pain, dyspnea, or non coronary chest pain. The symptomatic status did not seem to impact outcome, as the presence or absence of symptoms was not predictive of MACE and mortality. In comparison to symptomatic, asymptomatic patients were younger (age $63 \pm 11$, vs. $66 \pm 10, p=0.001$ ) and had worse resting echocardiography characteristics, with lower LVEF and higher WMSI $(52 \pm 13$ vs. $55 \pm 10, \mathrm{p}=0.008$; and $1.33 \pm 0.34$ vs. $1.22 \pm 0.31$, $\mathrm{p}=0.002$, respectively). However, the ischemic burden was lower in asymptomatic patients, with lower $\Delta$ in WMSI $(0.12 \pm 0.27$ vs. $0.19 \pm 0.29, \mathrm{p}=0.006$ ), and lower percentage of patients with clinical symptoms during ExE (17\% vs. 36\%, p<0.001) or with ECG positivity ( $15 \%$ vs. $25 \%, \mathrm{p}=0.01$ ). Accordingly, the performance of revascularizations within 6 months of the ExE was also lower in the asymptomatic group ( $5 \%$ vs. $16 \%$, p=0.001). During follow-up there were 92 MACE in the symptomatic group (21\% of the cases) and 40 MACE in the asymptomatic group (29\% of the cases), although the annualized MACE rate was the same for both groups (4.8\%). Also, independent predictors of MACE in the asymptomatic group were the same than in the overall group of patients: resting LVEF (HR=0.97, 95\% CI=0.05-1.00, p=0.05), maximal achieved workload in METs ( $\mathrm{HR}=0.82,95 \% \mathrm{CI}=0.73-0.93, \mathrm{p}=0.001)$, and ischemia on $\operatorname{ExE}(\mathrm{HR}=1.12,95 \% \mathrm{CI}=1.12-4.13, \mathrm{p}=0.02)$.

\section{Revascularizations during follow-up}

During follow up, there were 166 revascularizations (29\%) for clinical reasons (154 by a percutaneous approach and 12 by a repeated surgery). A total of 75 revascularizations were performed within 180 days of the ExE. Patients who underwent these earliest revascularizations had more frequently ischemic results than those who did not go to revascularization or that went to a more delayed revascularization (ischemia on ExE, 91\% vs. 46\%; symptoms during exercise testing, $59 \%$ vs. $27 \%$; positive exercise ECG, $47 \%$ vs. $19 \%$; all $\mathrm{p}<0.001$ ). On the contrary non-early revascularized patients were more frequently asymptomatic at the time of the ExE ( $27 \%$ vs. $9 \%$, p $<0.001$ ), had more frequently fixed WMAs ( $57 \%$ vs. $43 \%, p=0.02$ ), and their resting LVEF was slightly lower ( $54 \pm 11$ vs. $56 \pm 8, \mathrm{p}=0.05$ ).

\section{Discussion}

The main findings of this study are that ExE results add significant prognostic information in symptomatic or asymptomatic patients with surgical coronary revascularization, either for MACE or for overall mortality. Ischemia was an independent predictor of events, even when other important and powerful variables extracted from the "exercise part of the ExE study" were taken into consideration, like maximal workload. Maximal workload would not have been possible to take into account if other types of stress were performed, like pharmacological stress echocardiography or pharmacological myocardial perfusion imaging. For the same reason the robustness of imaging seems to be higher when exercise is not feasible or not performed and these variables are not available. Our approach consisted of exercise on the treadmill with imaging acquisition while the patients were still exercising. We have previously demonstrated that this method has higher sensitivity for assessing ischemia $[12,16]$. This likely explains the high number of positive results. To do this, we have employed exercise protocols adjusted to the baseline characteristics of the patients. Although exercise is the method of stress recommended in guidelines for the assessment of patients with confirmed or suspected CAD $[6,17]$, to our knowledge, the role of exercise echocardiography in patients with prior CABS has been tested in just a few studies [3,4]. In one of them, exercise was marginally analyzed as most of the patients underwent a pharmacological stress echocardiogram with dypiridamole [3]. In that study peak wall motion score index increased the prediction of the model for outcome, although ischemia was not specifically addressed. In another one, the lack of increase of LVEF with exercise, as a surrogate of ischemia, was an independent predictor of cardiac events [4]. In line with our results, a lack of increase in LVEF was a robust marker of risk in our patients with annualized event rates higher that 7\%, the other important marker was extensive ischemia with annualized event rates of almost $10 \%$. Finally, although the reach of ExE in asymptomatic patients seemed to be more limited than in symptomatic, as the former had less ischemia burden, and subsequent revascularizations were consequently less performed, the true is that imaging also offered prognostic information in this group.

\section{Limitations}

The retrospective nature of the study must be recognized as a limitation for some of the analysis performance. Also, we have included only patients able to exercise. Although most of our patients with CABS were able to exercise with adjusted protocols, our data could not be the same for patients with a theoretically higher risk, as those with inability to exercise. Finally, these patients were studied during a long-term period. Indications for testing could have changed during this period, as well as imaging technology and the kind of surgery employed. For instance, over the years, arterial grafts and off-pump surgery have been more frequently used.

\section{Clinical Implications}

Ischemia during exercise is frequent in patients with previous CABS, and is predictive of major cardiac events. Exercise 
echocardiography revealed twice-fold events in patients with ischemia and fourth-fold events in patients with abnormal ExE results, in comparison to patients without these characteristics. ExE should be considered as a first line tool for evaluation after CABS.

\section{References}

1. Harb SC, Marwick TH (2014) Prognostic value of stress imaging after revascularization: a systematic review of stress echocardiography and stress nuclear imaging. Am Heart J 167: 77-85.

2. Cortigiani L, Sicari R, Bigi R, Gherardi S, Rigo F, et al (2008) Usefulness of stress echocardiography for risk stratification of patients after percutaneous coronary intervention. Am J Cardiol 102: 1170-1174.

3. Cortigiani L, Bigi R, Sicari R, Landi P, Bovenzi F, et al. (2010) Stress echocardiography for the risk stratification of patients following coronary bypass surgery. Int J Cardiol 143: 337-342.

4. Arruda AM, McCully RB, Oh JK, Mahoney DW, Seward JB (2001) Prognostic value of exercise echocardiography in patients after coronary artery bypass surgery. Am J Cardiol 87: 1069-1073.

5. Pandey A, McGuire DK, de Lemos JA, Das SR, Berry JD, et al. (2016) Revascularization Trends in Patients With Diabetes Mellitus and Multivessel Coronary Artery Disease Presenting With Non-ST Elevation Myocardial Infarction: Insights From the National Cardiovascular Data Registry Acute Coronary Treatment and Intervention Outcomes Network Registry-Get with the Guidelines (NCDR ACTION RegistryGWTG). Circ Cardiovasc Qual Outcomes 9: 197-205.

6. Wolk MJ, Bailey SR, Doherty JU, Douglas PS, Hendel RC, et al. (2014) ACCF /AHA /ASE /ASNC/ HFSA/ HRS /SCAI /SCCT/ SCMR/ STS 2013 multimodality appropriate use criteria for the detection and risk assessment of stable ischemic heart disease: a report of the American College of Cardiology Foundation Appropriate Use Criteria Task Force, American Heart Association, American Society of Echocardiography, American Society of Nuclear Cardiology, Heart Failure Society of America, Heart Rhythm Society, Society for Cardiovascular Angiography and Interventions, Society of Cardiovascular Computed Tomography, Society for Cardiovascular Magnetic Resonance, and Society of Thoracic Surgeons. J Am Coll Cardiol 63: 380-406.

7. Arós F, Boraita A, Alegría E, Alonso AM, Bardají A, et al. (2000) Guidelines of the Spanish Society of Cardiology for clinical practice in exercise testing. Rev Esp Cardiol 53: 1063-1094.
8. Bouzas-Mosquera, Peteiro J, Alvarez-Garcia N, Broullon FJ, Mosquera VX, et al. (2009) Prediction of mortality and major cardiac events by exercise echocardiography in patients with normal exercise electrocardiographic testing. J Am Coll Cardiol 53: 1981-1990.

9. Peteiro J, Garrido I, Monserrat L, Aldama G, Calviño R, et al. (2004) Comparison of peak and postexercise treadmill echocardiography with the use of continuous harmonic imaging acquisition. J Am Soc Echocardiogr 17: 1044-1049.

10. Peteiro J, Bouzas-Mosquera A, Broullón FJ, Garcia-Campos A, Pazos P, et al. (2010) Prognostic value of peak and post-exercise treadmill exercise echocardiography in patients with known or suspected coronary artery disease. Eur Heart J 31: 187-195.

11. Lang RM, Badano LP, Mor-Avi V, Afilalo J, Armstrong A, et al. (2015) Recommendations for cardiac chamber quantification by echocardiography in adults: an update from the American Society of Echocardiography and the European Association of Cardiovascular Imaging. J Am Soc Echocardiogr 28: 1-39.

12. Hoffmann R, Lethen H, Marwick T, Rambaldi R, Fioretti P, et al. (1998) Standardized guidelines for the interpretation of dobutamine echocardiography reduce interinstitutional variance in interpretation. Am J Cardiol 82: 1520-1524.

13. Stamm RB, Carabello BA, Mayers DL, Martin RP (1982) Two-dimensional echocardiographic measurement of left ventricular ejection fraction: prospective analysis of what constitutes an adequate determination. Am Heart J 104: 136-144.

14. Lauer MS, Blackstone EH, Young JB, Topol EJ (1999) Cause of death in clinical research: time for a reassessment? J Am Coll Cardiol 34: 618620.

15. Hachamovitch R, Di Carli MF (2008) Methods and limitations of assessing new noninvasive tests: Part II: Outcomes-based validation and reliability assessment of noninvasive testing. Circulation 117: 2793-2801.

16. Peteiro J, Bouzas-Mosquera A, Estevez R, Pazos P, Piñeiro M, et al. Headto-head comparison of peak supine bicycle exercise echocardiography and treadmill exercise echocardiography at peak and at post-exercise for the detection of coronary artery disease. J Am Soc Echocardiogr 25: 319-326.

17. Pellikka PA, Nagueh SF, Elhendy AA, KuehlCA, SawadaSG (2007) American Society of Echocardiography. American Society of Echocardiography recommendations for performance, interpretation, and application of stress echocardiography. J Am Soc Echocardiogr 20:1021-1041.

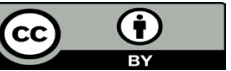

This work is licensed under Creative Commons Attribution 4.0 License

Submission Link: http://biomedres.us/submit-manuscript.php

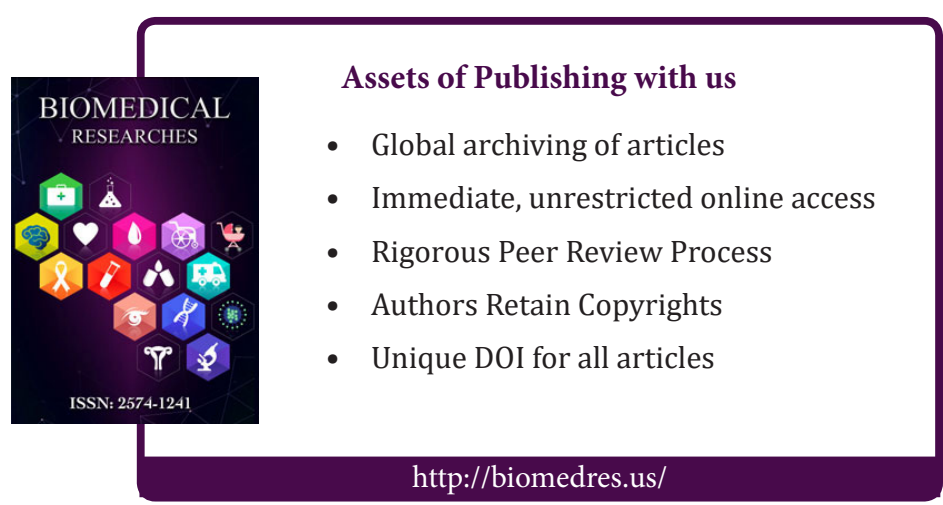

